\title{
Stabilization of a high-order harmonic generation seeded extreme ultraviolet free electron laser by time-synchronization control with electro-optic sampling
}

H. Tomizawa ${ }^{1}$, T. Sato ${ }^{1}$, K. Ogawa ${ }^{1}$, K. Togawa ${ }^{1}$, T. Tanaka ${ }^{1}$, T. Hara ${ }^{1}$, M. Yabashi ${ }^{1}$, H. Tanaka ${ }^{1}$, T. Ishikawa ${ }^{1}$, T. Togashi ${ }^{2}$, S. Matsubara ${ }^{2}$, Y. Okayasu ${ }^{2}$, T. Watanabe ${ }^{2}$, E.J. Takahashi ${ }^{3}$, K. Midorikawa ${ }^{3}$, M. Aoyama ${ }^{4}$, K. Yamakawa ${ }^{4}$, S. Owada ${ }^{5}$, A. Iwasaki ${ }^{5}$, and K. Yamanouchi ${ }^{5}$

${ }^{1}$ RIKEN, SPring-8 Center, Kouto 1-1-1, Sayo, Hyogo 679-5148, Japan

${ }^{2}$ Japan Synchrotron Radiation Research Institute, Kouto 1-1-1, Sayo, Hyogo 679-5198, Japan

${ }^{3}$ RIKEN Center for Advanced Photonics, Hirosawa 2-1, Wako, Saitama 351-0198, Japan

${ }^{4}$ Japan Atomic Energy Agency, Umemidai 8-1-7, Kizugawa, Kyoto 619-0215, Japan

${ }^{5}$ The University of Tokyo, Hongo 7-3-1, Bunkyo-ku, Tokyo 113-0033, Japan

(Received 14 January 2015; revised 10 March 2015; accepted 2 April 2015)

\begin{abstract}
A fully coherent free electron laser (FEL) seeded with a higher-order harmonic (HH) pulse from high-order harmonic generation (HHG) is successfully operated for a sufficiently prolonged time in pilot user experiments by using a timing drift feedback. For HHG-seeded FELs, the seeding laser pulses have to be synchronized with electron bunches. Despite seeded FELs being non-chaotic light sources in principle, external laser-seeded FELs are often unstable in practice because of a timing jitter and a drift between the seeding laser pulses and the accelerated electron bunches. Accordingly, we constructed a relative arrival-timing monitor based on non-invasive electro-optic sampling (EOS). The EOS monitor made uninterrupted shot-to-shot monitoring possible even during the seeded FEL operation. The EOS system was then used for arrival-timing feedback with an adjustability of $100 \mathrm{fs}$ for continual operation of the HHG-seeded FEL. Using the EOS-based beam drift controlling system, the HHG-seeded FEL was operated over half a day with an effective hit rate of 20\%-30\%. The output pulse energy was $20 \mu \mathrm{J}$ at the $61.2 \mathrm{~nm}$ wavelength. Towards seeded FELs in the water window region, we investigated our upgrade plan to seed high-power FELs with HH photon energy of 30-100 eV and lase at shorter wavelengths of up to $2 \mathrm{~nm}$ through high-gain harmonic generation (HGHG) at the energy-upgraded SPring-8 Compact SASE Source (SCSS) accelerator. We studied a benefit as well as the feasibility of the next HHG-seeded FEL machine with single-stage HGHG with tunability of a lasing wavelength.
\end{abstract}

Keywords: arrival-timing monitor; electro-optic sampling (EOS); high-gain harmonic generation (HGHG); high-order harmonic generation (HHG); self-amplified spontaneous emission (SASE); the soft x-ray water window region; wavelength-tunable seeded FEL; X-ray free electron laser (XFEL)

\section{Introduction}

A free electron laser (FEL) was proposed as one of the most promising coherent light sources with arbitrary wavelength in $1971^{[1]}$. Today, using self-amplified spontaneous emission (SASE) schemes, FELs are available in a wide region up to hard $\mathrm{x}$-rays ${ }^{[2]}$. The SPring-8 Compact SASE Source

Correspondence to: H. Tomizawa, RIKEN, SPring-8 Center, Kouto 1-1-1, Sayo, Hyogo 679-5148, Japan. Email: hiro@ spring8.or.jp
$(\mathrm{SCSS})^{[3]}$, which is a prototype FEL machine at SPring-8, generates extreme ultraviolet (EUV) pulses at wavelengths from 50 to $60 \mathrm{~nm}$. The SCSS was constructed for feasibility tests of new components to embody our x-ray FEL (XFEL) machine concept of the SPring-8 Angstrom Compact Free Electron Laser (SACLA) ${ }^{[4]}$. We are upgrading this EUVFEL to a higher energy machine with the aim of lasing at even shorter wavelengths. It consists of a high-brightness thermionic electron gun with several bunching sections, a high-energy accelerator and in-vacuum undulators. 


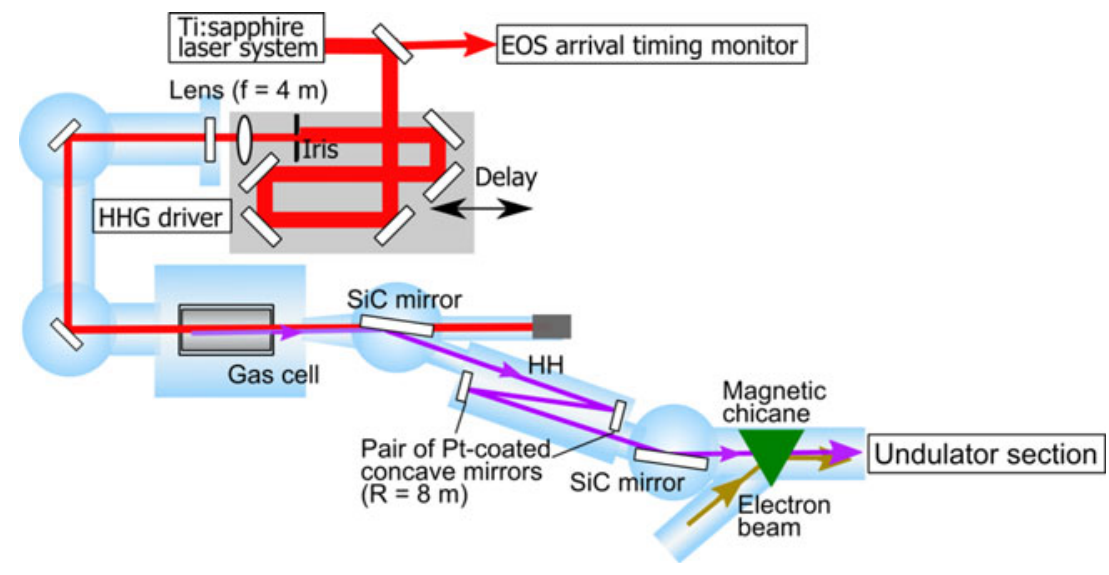

Figure 1. Schematic drawing of HHG and its beam transportation system for a seeded EUV-FEL. HH pulses are generated in a xenon gas cell with a lens (focal length: $4 \mathrm{~m}$ ) and separated from the fundamental pulses of the Ti:S laser by the first $\mathrm{SiC}$ mirror. A pair of Pt-coated concave mirrors with an $8 \mathrm{~m}$ curvature radius is used for the loose focusing $\mathrm{HH}$ pulses. The seeding $\mathrm{HH}$ pulses are selectively reflected by the second $\mathrm{SiC}$ mirror, and fully overlap the electron bunch at the front-end of the first undulator (Undulator 1).

In the SASE scheme, spontaneous radiation is first generated at the front-end of the first undulator. Then, it is exponentially amplified up to the saturated intensity level in a single pass while travelling in the following undulators ${ }^{[5]}$. Due to this radiation mechanism, an SASE-FEL pulse is not temporally coherent. That is, the spectra have uncontrollable spikes in the frequency domain and fluctuate shot-to-shot. The fluctuation of SASE-FEL pulses makes interpretations of experimental results difficult. To provide a spectrum with a targeted single peak on demand for user experiments, a fully coherent seeding source is required without an optical cavity in shorter wavelengths below the EUV region.

To solve this problem, a number of seeding schemes have been proposed up to now. Seeding schemes are roughly categorized into two kinds of approach, those that use some external seed sources and those that use absolutely none. One is called self-seeding, in which an SASE pulse monochromatized just after the first undulator section is injected into the second undulator section as the seeding source $^{[6,7]}$. In the other scheme, an external optical laser pulse is independently provided and used as a fully coherent seeding source. Recently, the high-order harmonic generation (HHG) of a femtosecond laser pulse focused in a gaseous medium was expanded up to the water window region and became a practical fully coherent light source in this region ${ }^{[8]}$. It is possible to seed FELs in the EUV wavelength region. However, since the pulse energy is limited and is insufficient to overcome the shot noise at a shorter wavelength, the external seeding scheme becomes much more difficult. To extend FEL seeding to a shorter wavelength, the seeding scheme called high-gain harmonic generation $(\mathrm{HGHG})^{[9]}$ is often adopted. In the user facility at FERMI (Elettra), the third-order harmonic (TH) pulse of a Ti:sapphire (Ti:S) laser is used as an optical seeder and generates the seeded FEL at its 13th harmonic $(20 \mathrm{~nm})$ with single-stage $\mathrm{HGHG}^{[10]}$ and furthermore at the fifth harmonic $(4 \mathrm{~nm})$ of the first HGHG pulse with a double cascade of $\mathrm{HGHG}^{[11]}$. In this system, the seeding TH pulse $(\sim 260$ $\mathrm{nm}$ ) is generated with a nonlinear crystal because of higher conversion efficiency. This allows the utilization of an optical seeder with a high pulse energy of the millijoule level, but a longer wavelength far from the final target wavelength of lasing.

In the hard x-ray region, self-seeding has been established as a robust and promising method. However, for the wavelength region longer than soft x-rays, self-seeding seems not to be so simple. In SPring-8, we made a decision to select the feasible self-seeding utilizing Bragg reflection from a thin diamond crystal $^{[7]}$ as the main seeding plan at SACLA for hard X-rays, and an HHG-based seeder for soft x-rays as an optional plan at SCSS. By directly seeding in the EUV region we developed an external seeding system with higherorder harmonic $(\mathrm{HH})$ pulses from 50 to $62 \mathrm{~nm}$ (Figure 1). These correspond to the 13 th $(61.5 \mathrm{~nm})$ and 15 th $(53.3 \mathrm{~nm})$ harmonics of a Ti:S laser (central wavelength: $800 \mathrm{~nm}$ ). In 2010, we achieved the first successful seeding of the SCSS EUV-FEL at the 13th harmonic of the Ti:S laser ${ }^{[12]}$. However, the low contrast ratio of the pulse energy of the seeded FEL pulses with respect to the SASE background noise as well as the low probability of successful seeding events were not favoured for user experiments. In addition, the best seeding conditions did not last more than ten minutes. The main reason for the small number of successful shots of the seeded FEL pulses was the poor temporal overlap between the 13th-order harmonic pulses and the electron bunches at the undulator. By applying a relative arrivaltiming monitor based on electro-optic sampling (EOS) for the timing feedback, we improved the beam parameter setup and HHG seeding performance at SCSS. As a result, we observed a clear difference between the SASE-FEL and a seeded FEL with a high contrast. 
In this paper, improvements in the $\mathrm{HH}$-seeding quality at the SCSS EUV-FEL are described in detail. By applying a non-invasive EOS arrival-timing monitor with feedback, sufficient FEL pulses are continuously seeded in a narrow spectral band. In this first stage of our development, we choose direct HHG seeding to study the feasibility of an HGHG scheme started from the shortest possible HH wavelength. In the next stage, to extend the FEL photon energy to the water window region with wavelength tunability, we propose single-stage HGHG with continuum HHG by mixing multicycle two-colour laser fields. To reduce the noise degradation of seeding, the total harmonic number of HGHG should be as low as possible. Therefore, we do not choose a multistage HGHG scheme in order to avoid noise degradation due to the frequency multiplication process $^{[13]}$.

\section{Setup of HHG direct seeding at SCSS}

Our machine setup of the HHG-seeded FEL is shown in Figures 1 and 2. It consists of an accelerator equipped with a variety of beam monitors to optimize overlapping conditions, and an ultrafast optical laser source. HH pulses from a femtosecond Ti:S laser are generated in a xenon gas cell and transported into the undulator section. In the direct seeding scheme with an external $\mathrm{HH}$ laser pulse, maximization of the overlap between the seed laser pulse and the electron bunch is crucial in 6D phase space $\left(x, x^{\prime}, y, y^{\prime}, t, E\right)$ at the frontend of the first undulator. Here, $x$ and $y$ are the horizontal and vertical centroid positions (sizes), $x^{\prime}$ and $y^{\prime}$ are their normalized momenta (divergences), and $t$ and $E$ are the time and the photon energy (central wavelength). The spatial overlap is checked with screen monitors at two points in the undulator section. Additionally, the EOS monitor checks the temporal overlap in real time. Utilizing the electrooptic (EO) probe pulse split from the HHG-driving laser pulse, the arrival time difference between the seeder pulse and the electron bunch is under control and is fixed at the optimal seeding condition with monitoring spectra and the pulse energy of the seeded FEL pulses.

\subsection{SCSS prototype accelerator and operation parameters for seeding}

The SCSS test accelerator consists of a thermionic electron gun, RF bunch compressors (lowest frequency of RF cavity: $238 \mathrm{MHz})$, a C-band (5712 MHz) main Linac and two invacuum undulators (each of $4.5 \mathrm{~m}$ length). Electron bunches are generated at the pulsed thermionic electron gun with a $\mathrm{CeB}_{6}$ single crystal cathode ${ }^{[14]}$ with a repetition rate of $30 \mathrm{~Hz}$. The electron bunches are mainly compressed through a velocity bunching process in the RF bunch compressor cavities and accelerated by a C-band high-gradient Linac up to $250 \mathrm{MeV}$. At the end, the beam is injected into a series of in-vacuum undulators with $15 \mathrm{~mm}$ period lengths and 300 undulator periods. For the HHG seeding operation, a bunch charge is set to $300 \mathrm{pC}$ and the transverse beam size is $\sim 200 \mu \mathrm{m}$ (FWHM) in a diameter at the entrance of the first undulator. One notable difference in the accelerator setup between SASE and seeded FEL operations is the electron bunch length. For SASE, the bunch length is normally adjusted to be the shortest so that the FEL gain is maximized. For a seeded FEL, the highest gain may not be the best. Since the seed pulse length provided by the 13th harmonic is of the order of tens of femtoseconds, i.e., shorter than the nominal electron bunch length in our case, SASE is inevitably amplified where the seed pulse does not exist in the electron bunch. In order to generate single-spike, fully coherent FEL pulses, it is necessary to suppress SASE amplification as much as possible, yet a seeded FEL needs to be above a certain level. The first requirement for this is to prepare a seed laser pulse with a peak power higher than the shot noise in the lethargy regime. In our case, HH laser pulses are able to provide enough peak power. Second, the electron bunch length should be somehow optimized so that the contrast ratio between the SASE and the seeded FEL is maximized. According to numerical simulations in our case, a bunch length of $1 \mathrm{ps}$ (FWHM) is too long with a lower peak current to obtain enough gain in a seeded FEL, while the nominal bunch length for SASE operations, $300 \mathrm{fs}$, is too short with a higher peak current to suppress SASE gain in our experimental conditions. As a result, it was a good compromise to fix the bunch length at $600 \mathrm{fs}$ (FWHM). Note that the longer bunch length also helps to mitigate the shotto-shot fluctuation of the output FEL power coming from the timing jitter between electron bunches and seed laser pulses.

The seeded FEL pulses are transported to the photon beamline of the SCSS EUV-FEL (Figure 2). During the seeding operations, we measure the spectra of the seeded FEL pulses with a single-shot EUV spectrometer, which has $10^{3}$ resolving power $(\lambda / \Delta \lambda)$ around $60 \mathrm{~nm}$, at the end of the photon diagnostics line. The pulse energy of the seeded FEL is detected by a gas monitor detector installed at the front-end of the beamline utilized by users. The gas monitor detector filled with argon gas has been calibrated with a calorie meter and it enables us to obtain the absolute pulse energy non-destructively ${ }^{[15]}$. These beamlines are switched by inserting a mirror at the entrance of the photon diagnostics line.

\section{2. $H H G$ driver and $E O$ probe pulses generated from the common laser source}

The external optical laser system for the driver of the HHG and the EOS probe pulse is based on a chirped pulsed amplification system of a femtosecond Ti:S laser $(800 \mathrm{~nm}$, $180 \mathrm{fs}, 30 \mathrm{~Hz})$. The system consists of a mode-locked oscillator (Spectra-Physics: Tsunami) that is synchronized to 


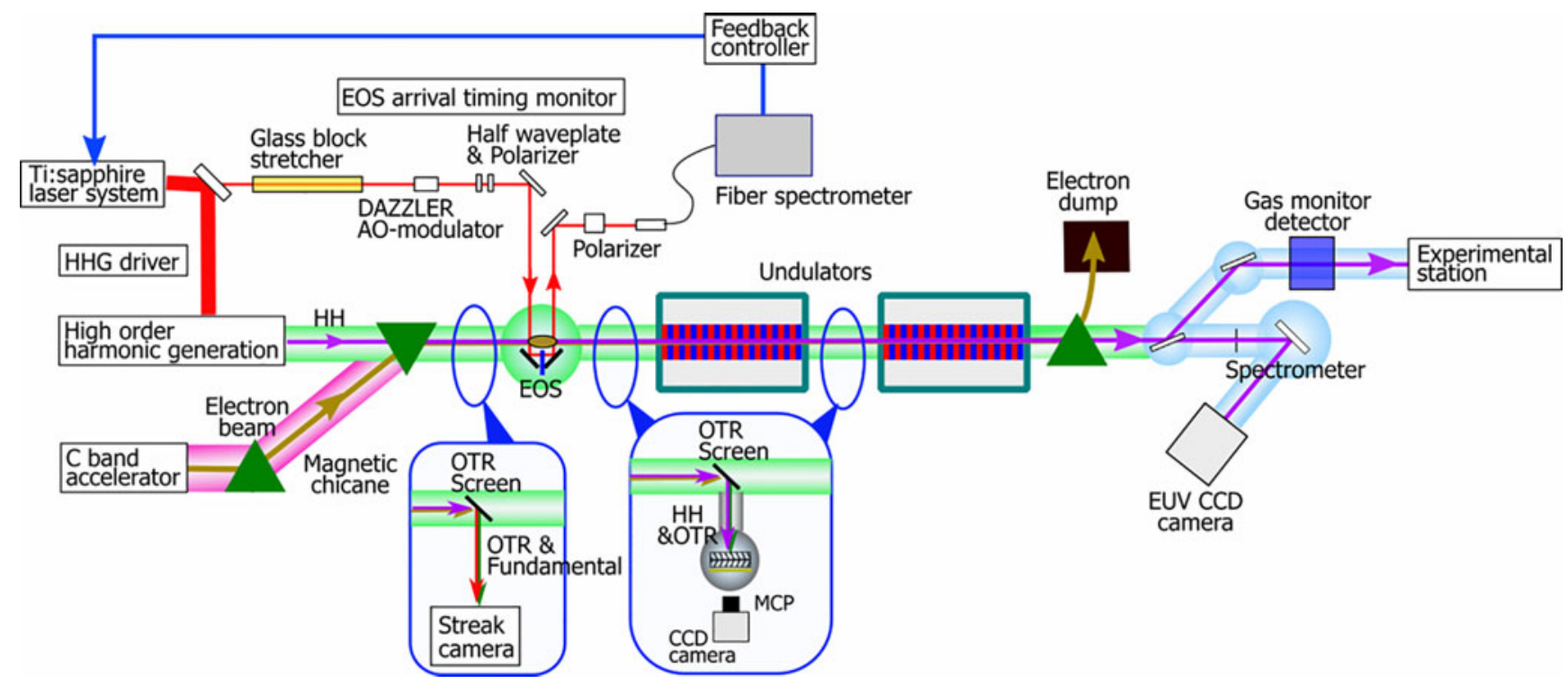

Figure 2. Schematic drawing of the HHG-seeded FEL system with a timing drift control. This seeded FEL system consists of an SCSS FEL machine (C-band accelerator, magnetic chicane and in-vacuum undulators), a Ti:S laser system (which is the common laser pulse source for an HHG seeding system) and an EOS-based arrival-timing monitor. The EOS arrival-timing monitor is installed before the first undulator. Utilizing the EO probe pulse optically split from the HHG-driving laser pulse, the arrival time difference of the seed laser pulse and the electron bunch is under control and is fixed at the optimal seeding condition. The spectra and pulse energy of the seeded FEL are measured with a single-shot spectrometer and a gas monitor detector at the end of the beamline, respectively. Insets: the spatial profile of the seeding pulse and the electron bunch on a phosphor screen are measured with microchannel plates at the entrance and end of the first undulator. Temporal overlap is roughly checked by a streak camera at sweep ranges from $1 \mathrm{~ns}$ down to $50 \mathrm{ps}$.

the $238 \mathrm{MHz}$ master clock of the SCSS by feedback locking the cavity length, a regenerative amplifier (Spectra-Physics: Spitfire) and a home-built four-pass amplifier. The output pulse energy is up to $120 \mathrm{~mJ}$ at the laser source and $30 \%$ of this output is used for the seeding operation. More than $99 \%$ of the pulse energy is used for the HHG system, and the residual energy for the probe pulse of EOS. The EO probing pulse is stretched by passing through a bulk stretcher before arriving at the EO crystal. It gives enough dynamic range to measure the timing drift of the electron bunch with respect to the $\mathrm{HH}$ pulse. For FEL seeding with an external laser pulse, it is essential to establish the coincidence and to sustain the overlapping condition between the $\mathrm{HH}$ pulses and electron bunches.

On the other hand, the HHG-driving laser pulse with a pulse energy of $30 \mathrm{~mJ}$ is loosely focused by a plano-convex lens (focal length: $4 \mathrm{~m}$ ) and delivered into the target chamber through a thin window. We set the focus around the entrance pinhole of an interaction cell that is filled with xenon gas. The target gas pressure is adjusted to balance the geometrical phase shift and the harmonic dipole phase. The HH pulse is selectively reflected with $\mathrm{SiC}$ mirrors set at the Brewster angle $\left(69^{\circ}\right)$ to allow the fundamental Ti:S laser through. By introducing a pair of Pt-coated, nearly normal-incidence mirrors with $8 \mathrm{~m}$ curvature radii, the $\mathrm{HH}$ pulses are loosely focused in the front-end of the first undulator.

After being reflected at the second $\mathrm{SiC}$ separator mirror, the $\mathrm{HH}$ pulse is combined with the electron beam at the magnetic chicane, and then both beam are introduced to the undulator section. Since the SiC mirrors reflect EUV light above $30 \mathrm{~nm}$, a few orders of $\mathrm{HH}$ including the 13th are selected and sent to the undulator. The beam diameter of the 13th harmonic at the entrance of the first undulator is $\sim 500 \mu \mathrm{m}$ (FWHM). The pulse energy of the 13th harmonic is estimated to be $2 \mathrm{~nJ}$, which is measured downstream and calibrated by the spectrometer as well as the gas monitor detector at the end station. The total optical throughput of the $\mathrm{HH}$ laser transportation is $\sim 1 \%$ (the $\mathrm{HH}$ pulse is generated with $\sim 200 \mathrm{~nJ}$ at the gas cell). The resulting peak power is estimated to be $40 \mathrm{~kW}$ in the seeding region, assuming a pulse duration of 50 fs (FWHM).

\subsection{Measurement to keep overlapping between electron bunches and $\mathrm{HH}$ pulses}

To optimize the seeding conditions, the $\mathrm{HH}$ pulses and electron bunches must overlap each other in the 6D phase space. Thus, beam diagnostics for achieving optimal 6D overlapping are key requirements for successful operation. To obtain a sufficient power gain of the FEL amplifier and overcome the shot noise under the restriction of seeding pulse energy, both the $\mathrm{HH}$ pulse and the electron bunch are compressed in the $6 \mathrm{D}$ phase space volume as much as possible. However, this makes it difficult to keep the overlapping condition continuously. Therefore, we measure the temporal and spatial sizes of both the electron bunches and $\mathrm{HH}$ pulses at the first undulator and relax the overlapping 


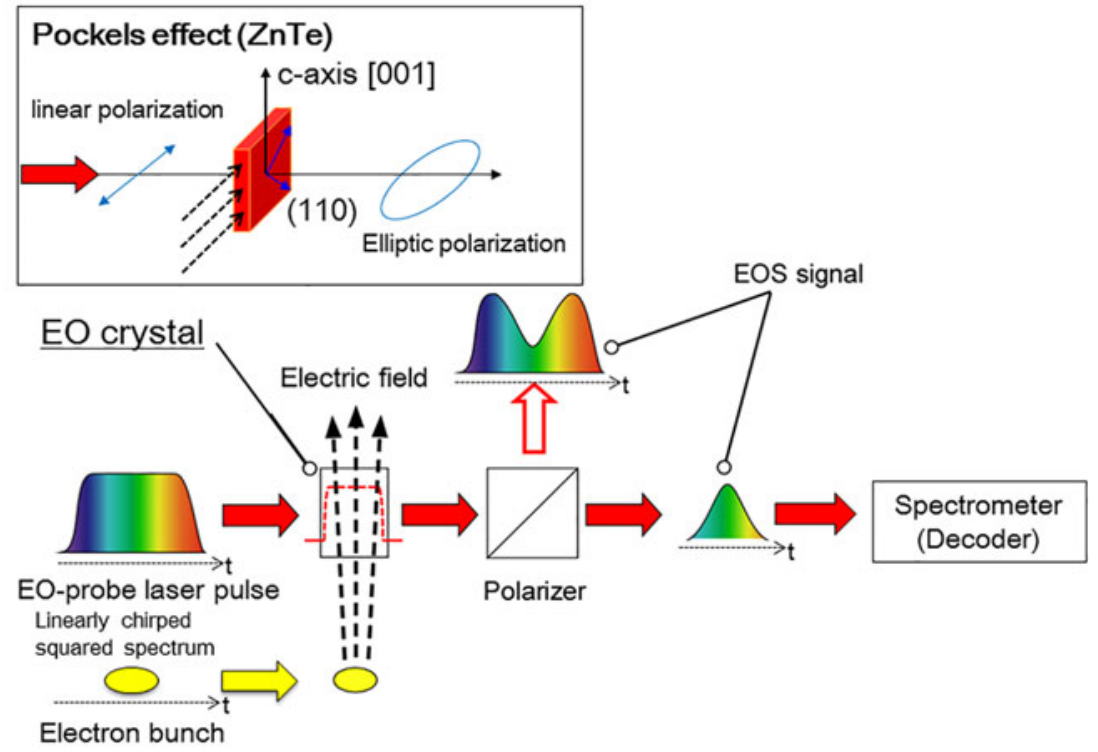

Figure 3. Principle of EOS measurement in the manner of spectral decoding. The probe pulse is linearly chirped and acts as a carrier wave for spectral decoding EOS. In addition, the use of a linear-chirped laser pulse with a flat-top spectrum to probe an ultrafast EO crystal (ZnTe) makes it possible to characterize the temporal bunch charge distribution precisely in real time. The adaptive AO modulator (Fastlite: Dazzler HR45-650-1100) is able to shape both the spectral phase and the intensity distributions of laser pulses with broadband spectra. The EO crystal is set near the electron beam. The linear polarization of the carrier wave changes into an elliptic polarization mainly due to the Pockels effect in the EO crystal under the electric field of the electron bunch. The information on the electron bunch charge distribution is encoded as the intensity modulation in the spectrum, and decoded bunch-by-bunch by a multichannel spectrometer.

conditions by enlarging both the sizes as long as the seed power keeps a sufficiently high contrast against the shot noise of the electron beam.

The spatial profiles and positions of the seeding pulse and the electron bunch are measured on the same area sensor cameras composed of microchannel plates (MCPs) with phosphor screens at the entrance and the end of the first undulator. The system allows simultaneous monitoring of the spatial profiles of the $\mathrm{HH}$ pulse and the optical transition radiation (OTR) from the electron beam to ensure spatial overlapping while travelling in the first undulator. The mismatch of the transverse centroid position and the angle of the direction between the $\mathrm{HH}$ pulses and the electron bunches are suppressed into ranges of less than $100 \mu \mathrm{m}$ and $100 \mu \mathrm{rad}$ by precisely steering the optical path of the $\mathrm{HH}$ pulse using the two Pt-coated mirrors.

On the other hand, temporal overlapping is also crucial for the seeding scheme. For this purpose, sequential tuning steps combining fine and coarse adjustments are applied. First, the timing difference between the OTR of the electron bunch and the HHG-driving Ti:S laser pulse is roughly measured with a streak camera (Hamamatsu Photonics K.K: FESCA-200). Then the timing difference is adjusted with an electrical delay unit (Candox Systems Inc.: 84DgR238B01) of the Ti:S laser, and consequently lowered to a certain extent ( $<1 \mathrm{ps})$. Finally, the peak positions of the EO signals are kept at the same wavelength as the optimal seeding condition which is defined with the monitoring spectra and pulse energy of the seeded FEL pulses downstream.

\subsection{Arrival-timing measurement with EOS and feedback system}

In the relative arrival-timing measurement system, the EOS technique is applied in the manner of spectral decoding (Figure 3). It consists of a probe laser, an EO crystal, a polarizer and a multichannel spectrometer for real-time measurements. We use a zinc telluride (ZnTe (110): $3 \times$ $4 \mathrm{~mm}^{2}$ area and $1 \mathrm{~mm}$ thick) as the EO crystal in this setup. The $\mathrm{ZnTe}$ crystal is set in the vicinity of the electron beam path, in which the probe laser pulse is passing and modulated. The probe pulse is linearly chirped and works as a carrier wave with a linear relation between frequency (wavelength) and time (relative arrival timing). When the electron bunch passes near the EO crystal, the orthogonal polarization components of the EO probe pulse are retarded, one against the other (birefringence effect), through the crystal under the high electric field of the electron bunch. Then the polarization modulation is converted to intensity modulation in the spectrum by the polarizer. As a result, the electron bunch timing with respect to the EO probe laser pulse is encoded as the intensity modulation in the spectrum, and is diagnosed by a multichannel spectrometer in real time. The relative timing drift is compensated with a feedback control.

In our EOS system, an EO probe laser pulse is optically split from the common laser source to drive the HHG in the accelerator tunnel. After splitting, the EO-probe pulse is primarily stretched through a bulk stretcher made of 

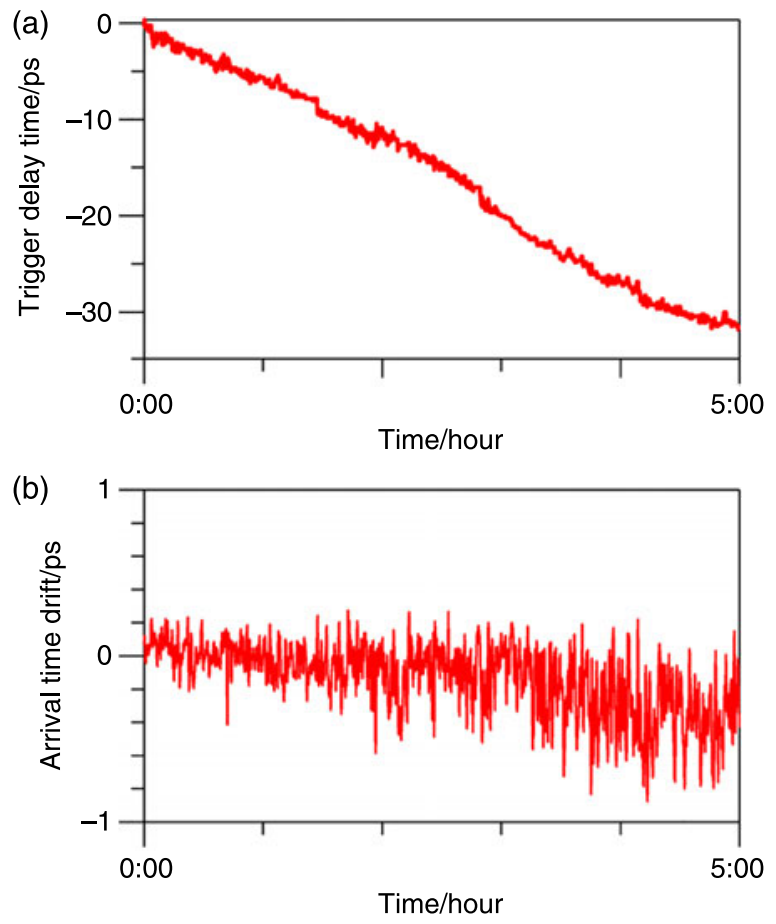

Figure 4. (a) The trigger delay time added to the Candox delay unit by using the home-built feedback system. (b) The relative arrival time of the electron bunch with respect to the optical laser pulse. Both (a) and (b) were measured for $5 \mathrm{~h}$ simultaneously.

high-index glass blocks (Ohara: S-NPH3). Next, to shape the spectrum flat-top and control the fine stretching factor with compensating non-linearity of the chirp, we install an adaptive AO modulator (Fastlite: Dazzler HR45-650-1100) as a fine adjustment tool. The arrival timing of the electron bunch with respect to the HHG driver laser pulse is decoded as the spectral peak of the EO signal. The EO signals are decoded by a multichannel spectrometer (Ocean Optics: QE65000). The relative timing between the $\mathrm{HH}$ pulse and the electron bunch is fixed at the optimal timing with a feedback system.

The delay time shown in Figure 4(a) is added to the Candox delay unit for five hours. As a consequence, the timing drift is suppressed, as shown in Figure 4(b). If the trigger delay had not been optimized by our feedback system, the relative arrival time drift that corresponds to the delay time shown in Figure 4(a) would have been observed.

\section{Experimental results and evaluation}

\subsection{Experimental results for the seeded FEL pulses}

Figure 5 compares typical spectra of the seeded FEL (red line) and the SASE (blue line) which was measured by the single-shot EUV spectrometer at the end of the beamline (shown in Figure 2). The spectrum of the seeded FEL has almost a single peak with a bandwidth of about $0.06 \mathrm{~nm}$

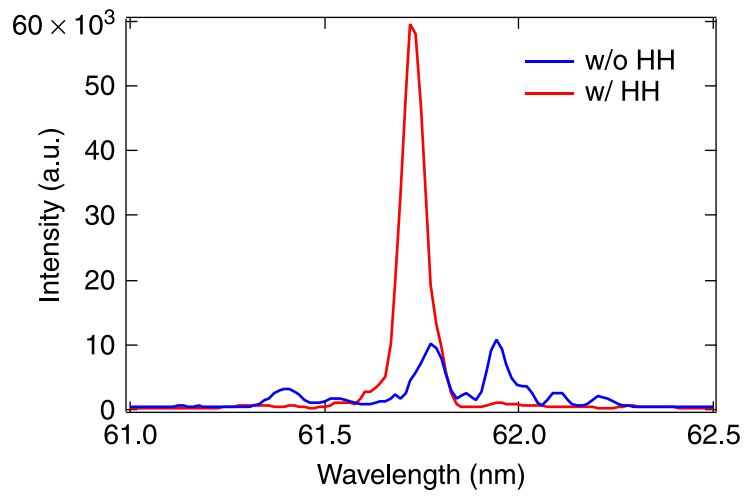

Figure 5. Comparison of the typical spectra of FEL pulses with (red line) and without (blue line) seeding HH pulses. The spectral bandwidth of the seeded FEL pulse was $0.06 \mathrm{~nm}$ (FWHM).

(FWHM). It follows that the bunch length of $600 \mathrm{fs}$ was appropriate for providing users with single-spike, fully coherent FEL pulses. With the existing setup, however, not all the output FEL pulses came in such ideal FEL pulses with a single spike; some distributions with many spikes were also observed in some shots. Thus, we evaluated the success rate of the seeding by introducing the following criteria.

For a quantitative evaluation of the probability of a successful seeding effect, we shall define an 'effective' hit rate. When the spectral peak intensity of the output pulse exceeded the threshold indicated in the following formula, we defined the event as an 'effective' hit:

$$
I_{\text {seed }}-\overline{I_{\mathrm{SASE}}}>4 \sigma_{\mathrm{SASE}}
$$

where $I_{\text {seed }}$ and $\overline{I_{\text {SASE }}}$ are the peak intensity with an $\mathrm{HH}$ pulse injected and the averaged peak intensity without the seeding source, respectively, and $\sigma_{\mathrm{SASE}}$ is the standard deviation of the statistical fluctuation without the seeding source. In the case of our former experiment in $2010^{[12]}$, according to this definition, the effective hit rate was calculated as $0.3 \%$.

Figure 6 shows the correlation data plot between the spectral peak intensity of the output pulse subtracted by the average SASE intensity and the wavelength of the peak intensity in the data for 10,000 shots with seeded FEL operation in 2012. In Figure 6, the red points represent the seeded pulses with effective hits, which exceeded $4 \sigma_{\text {SASE }}$. The blue points, which are less than $4 \sigma_{\mathrm{SASE}}$, are defined as ineffective hits. Spectral peak intensities above the threshold are distributed from 61.5 to $62.0 \mathrm{~nm}$. Conversely, the spectral peak intensities in the SASE-like region (blue points) are distributed over $2.5 \mathrm{~nm}$. The standard deviation of the peak intensity wavelength is $0.08 \mathrm{~nm}$ for the seeded FEL pulse with effective hits. Consequently, this statistical data analysis shows that the standard deviation is comparable to the spectral bandwidth of the seeded FEL pulses mentioned above. Our definition of the effective hit rate is useful to 


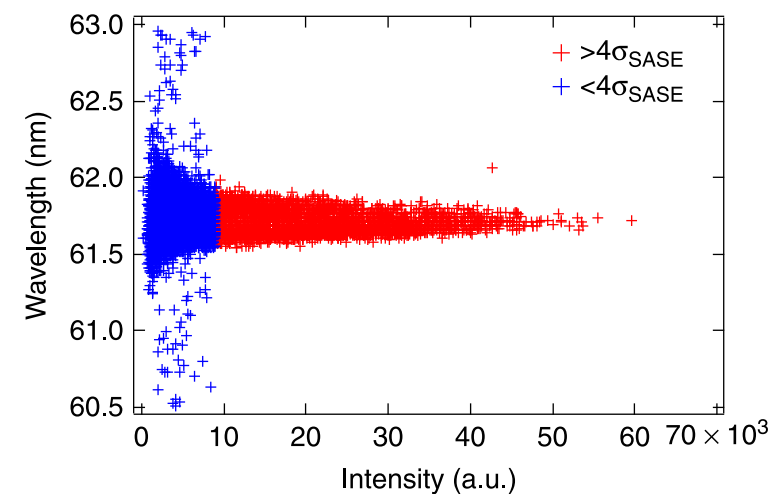

Figure 6. Correlation data plot between the normalized intensity and the spectral peak intensity with seeded operation. Here, $\sigma_{\text {SASE }}$ is the standard deviation of the peak intensities without the $\mathrm{HH}$ pulses under the seeding condition of the FEL. Effective seeded FEL pulses are defined as being large as $4 \sigma_{\mathrm{SASE}}(\mathrm{red})$ for our user experiments.

judge the seeding quality in our user experiments. For the 10,000 shot data in Figure 6, the effective hit rate was $30 \%$.

\subsection{Improvement of seeding condition with EOS feedback}

The trend graph of the peak intensity is shown in Figure 7. The ability to not only increase the hit rate but also sustain a seeding effect and keep the output power as stable as possible is critical to practical user experiments. As presented in Figure 7, the average output power was kept almost constant for hours while the pilot user experiment was being implemented, although shot-to-shot fluctuation was obviously an issue, as already discussed. In principle, the EO feedback system is able to keep the seeding effect as long as it is needed. It is also worth noticing that the sustained seeding is beneficial not only to experiments, but also to accelerator operations. In our former experimental result in $2010^{[12]}$, the output pulse energies and the seeded FEL gain were limited to $1.3 \mu \mathrm{J}$ and 650 , respectively. After applying the EOS feedback system, the output energy was increased up to 15-20 $\mu \mathrm{J}$, and the seeded FEL gain reached as much as $\sim 10^{4}$. Such an improvement was mainly due to the capability of the reproducible seeding condition in the time domain while adjusting other dimensions. For seeded FEL tunings, it is important to keep a temporal overlapping while the spatial and angular overlapping is being adjusted. Thus, the feedback system contributed not only to increasing the hit rate, but also to improving the contrast ratio of output intensities between the seeded FEL and SASE.

\section{Wavelength-tunable seeded FEL in the water window region of soft $x$-rays}

Here, we discuss further extension of the wavelength of a seeded FEL scheme to shorter wavelength regions. Despite

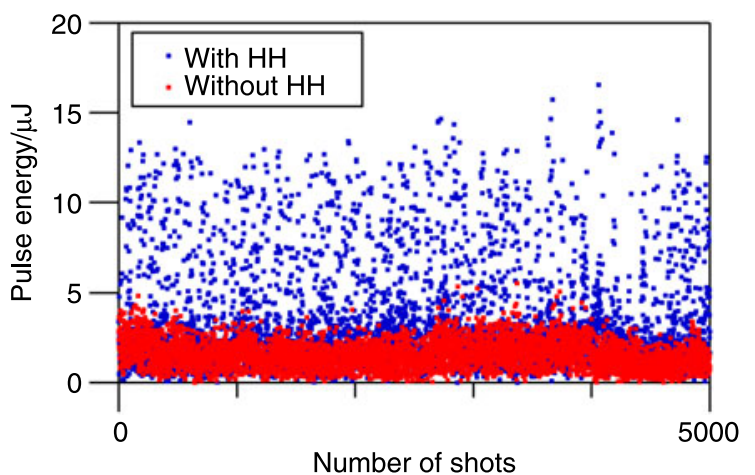

Figure 7. Trend graph of peak intensities of 5,000 FEL pulses in the seeded operations with (blue points) and without (red points) $\mathrm{HH}$ pulses. Experimental results of the experiment with feedback in 2012 are shown. The contrast ratio of the peak intensity was improved by a factor of $\sim 3$. In 2010, the seeded FEL pulse energy was $1.3 \mu \mathrm{J}^{[12]}$. During our experiments, we achieved a pulse energy of up to $20 \mu \mathrm{J}$ at maximum.

being one of the milestones of seeded FELs in the soft $\mathrm{X}$-ray region, seeded FELs have not been realized with a sufficient level of pulse energy for user experiments in the water window region.

Figure 8 shows the numerical simulation results for the output pulse energy after the FEL amplification versus the photon energy (radiation wavelength) in the case of singlestage HGHG assuming that the HH pulse energy is between 30 and $100 \mathrm{eV}$. To reduce the noise degradation of the seeding effect, we selected the shortest $\mathrm{HH}$ wavelength (highest photon energy) possible in its plateau region and reduced the total harmonic number of HGHG to as low as possible.

In this simulation, the normalized emittance, energy spread and peak current of the electron beam were fixed to be $0.8 \mathrm{~mm} \mathrm{mrad}(\mathrm{RMS}), 8 \times 10^{-5}$ (RMS) and $1.2 \mathrm{kA}$, respectively, while the beam energies were varied from 1.0 to $1.8 \mathrm{GeV}$. We assumed $5 \mathrm{~cm}$ period undulators with a fixed $K$-value of 3.2 for the modulator section, and $2.8 \mathrm{~cm}$ period undulators for the radiator section with the $K$-values being tuned from 1.06 to 2.99 according to the harmonic number. At the same time, the $\mathrm{HH}$ photon energy was assumed to be tuned from 30 to $100 \mathrm{eV}$, with a beam size of $100 \mu \mathrm{m}$ (RMS), pulse length of $5 \mathrm{fs}$ (FWHM) and maximum pulse energy of $15 \mathrm{~nJ}$. Note that the input pulse energy was reduced under certain conditions to avoid oversaturation in the modulator section.

The results of this simulation show clearly that the present single-HGHG scheme can readily be applied to the wavelength region below $2 \mathrm{~nm}$ with a pulse energy level of a few tens of microjoules, where an $\mathrm{HH}$ pulse with sufficiently high intensity ( $>20 \mathrm{~nJ} /$ pulse) has already been achieved ${ }^{[16]}$. For seeding in the soft $\mathrm{x}$-ray region, it is necessary to improve the total throughput of $\mathrm{HH}$ pulses by more than ten times. To improve the throughput of $\mathrm{HH}$ transportation, we designed the optics with Kirkpatrick-Baez mirrors $(>80 \%)$ and Ptcoated mirrors with 1 degree of incident angle $(>95 \%)$. This 


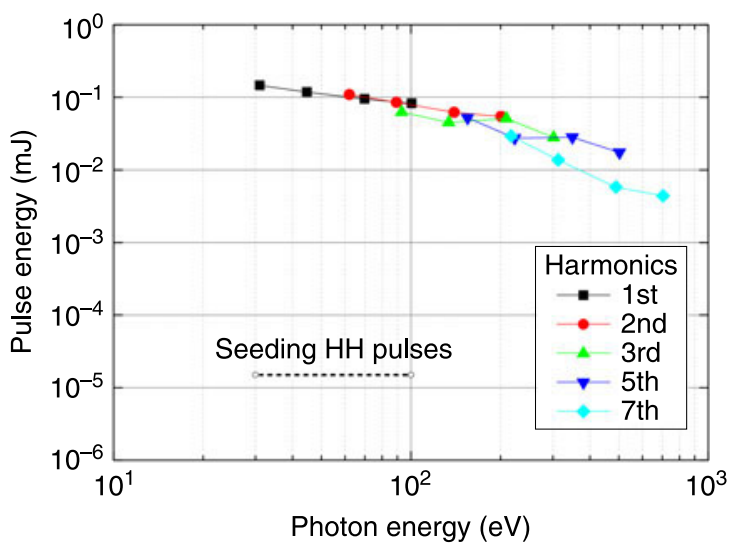

Figure 8. Expected pulse energies of the HHG-seeded HGHG FEL plotted as a function of the photon energy for different harmonic numbers. The harmonics of HGHG were calculated up to the seventh order. In this calculation, the photon energy of the seeding $\mathrm{HH}$ pulse was tuned from 30 to $100 \mathrm{eV}$, with a beam size of $100 \mu \mathrm{m}$ (RMS), pulse length of $5 \mathrm{fs}$ (FWHM) and maximum pulse energy of $15 \mathrm{~nJ}$. The conditions of the seeding $\mathrm{HH}$ pulses are feasible even in continuum HHG to realize a wavelength-tunable seeded FEL in the soft $\mathrm{x}$-ray water window region.

allowed us to obtain $15 \mathrm{~nJ}$ of a single harmonic pulse at the undulator.

We also considered that the seeded FEL in the water window region of soft x-rays with wavelength tunability is a useful light source for user experiments. Specifically, it is critically important to adjust the central wavelength of narrow-bandwidth seeded FELs for atomic, molecular and optical physics (AMO) experiments that aim at a specific wavelength for resonance absorptions. For wavelengthtunable operation, we proposed single-stage HGHG seeded with continuum HHG (high dense harmonics) generated by mixing multicycle two-colour laser fields with the fundamental $(800 \mathrm{~nm})$ and optical parametric amplifier $(1300 \mathrm{~nm})$ of a femtosecond Ti:S laser pulse ${ }^{[17]}$. By combining a twocolour field synthesis and an energy-scaling method of HHG, the isolated sub-femtosecond pulse reaches as high as $1.3 \mu \mathrm{J}$ at $\sim 30 \mathrm{eV}^{[18]}$. The bandwidths of individual $\mathrm{HH}$ pulses are wider than the bandwidth of SASE in the fundamental. According to our simulation, we can provide our users with a few tens of microjoules of seeded FEL at $500 \mathrm{eV}$. This seeding scheme is feasible with wavelength tunability and can be extended to be in the water window region.

\section{Summary and future plan}

By maintaining the best overlapping condition between the 50 fs $\mathrm{HH}$ pulse and the $600 \mathrm{fs}$ electron bunch, the 13th harmonic of a Ti:S laser was significantly amplified with the SCSS test accelerator employed as an FEL amplifier. The temporal and spatial overlaps of the electron bunch and the $\mathrm{HH}$ pulse, as well as the adjustment of the wavelength of the undulator radiation to that of the seeding $\mathrm{HH}$ pulse, were precisely tuned to achieve the seeded FEL operation.
Spectral narrowing was observed in our seeded conditions. Moreover, a relative arrival-timing monitor and a timing feedback system were introduced in this experiment. With the system, the seeded FEL performance was significantly improved over the long term. Compared with our former seeding condition in 2010, the pulse energy and the FEL gain of the seeded FEL were improved from 1.3 to $20 \mu \mathrm{J}$ and from 650 to $10^{4}$ (15 times improvement), respectively. The effective hit rate was improved from $0.3 \%$ to $20 \%-30 \%$ (improvement of two orders). Consequently, operation for pilot user experiments with the seeded FEL was attained at SCSS.

For an even higher effective hit rate rising towards to $100 \%$ in long-term operation, it is necessary to improve the homogeneity of the electron bunch charge density, the spectral flatness of the EOS probe laser and the relative pointing stability in the transverse overlapping. In order to maintain the best overlapping, the $\mathrm{HH}$ pulses and electron bunches must overlap each other in the 6D phase space. We adopted a strategy to overcome the uncontrollable timing and pointing jitter by covering it with large beam sizes. However, this strategy is guaranteed only in the case of a homogeneous electron bunch distribution and uniform current distribution within the overlapping region. In our seeding experiment, the electron bunch was de-bunched by up to $600 \mathrm{fs}$, but it seems that the effective longitudinal overlapping region was not longer than 400 fs according to scanning of the relative timing delay. The fine distribution of electron bunches needs to be further investigated to effectively suppress the jitter effects. To monitor the relative pointing to keep spatial overlap including their divergences, we are developing a single-shot 3D-BCD (three-dimensional bunch charge distribution) monitor at SPring- $8^{[19]}$. This extended EOS monitor and a sophisticated feedback system may suppress the long-term drift components of relative pointing stability. In this development, the EO probe laser has been developed as a lineally chirped broadband probe laser with a square spectrum with a large spectral flat-top range. By characterizing the nonlinear chirp of this EO probing laser pulse with a Dazzler-based chirp scanning measurement, we can finely adjust the pulse duration of an octave-broadband probe laser with a linear chirp rate of $1 \mathrm{fs} \mathrm{nm}^{-1}$ for probing EO crystals ${ }^{[19]}$. The octavebroadband flat-top spectrum of the EO probe laser gives not only the upper limit of temporal resolution, but also a large dynamic range for arrival timing (trends in timing drift and pointing data over time). It will be a great contribution to improving the seeded FEL operation with the sophisticated feedback system of EOS measurements.

In addition, further compression of the $\mathrm{HH}$ pulse under the restriction of the seeding pulse energy and the electron bunch is required for a single-stage HGHG aiming at the water window region. To achieve significant difference from the SASE, the seeding pulses must have the same wavelength as the resonance wavelength of the FEL with a 
power level well above the shot noise power. Accordingly, depending on the magnitude of the timing jitter, it becomes necessary to find an appropriate electron bunch duration with which the effective hit rate, the contrast ratio and the output power should be all in the acceptable ranges for the user experiments. With progress to even shorter wavelengths, the temporal resolution of EOS and the temporal response of EO crystal have to be improved to tens of femtoseconds (FWHM). For this purpose, we are developing a novel EOS system with the organic EO crystal DAST (4- $N, N$ dimethylamino- $4^{\prime}-N^{\prime}$-methyl stilbazolium tosylate) with a temporal response of $30 \mathrm{fs}$ (FWHM) ${ }^{[20,21]}$.

We seriously consider the generation of spatially and temporally coherent soft $\mathrm{x}$-rays by the proposed scheme as one of the candidates for our FEL upgrades. In order to enable a wavelength-tunable seeded FEL in the water window region of soft x-rays to work effectively and constantly, it is necessary to develop a high throughput of optics to transport $\mathrm{HH}$ pulses and to achieve a high conversion efficiency of continuum HHG due to a two-colour field synthesis.

\section{References}

1. J. M. J. Madey, J. Appl. Phys. 42, 1906 (1971).

2. P. Emma, R. Akre, J. Arthur, R. Bionta, C. Bostedt, J. Bozek, A. Brachmann, P. Bucksbaum, R. Coffee, F.-J. Decker, Y. Ding, D. Dowell, S. Edstrom, A. Fisher, J. Frisch, S. Gilevich, J. Hastings, G. Hays, Ph. Hering, Z. Huang, R. Iverson, H. Loos, M. Messerschmidt, A. Miahnahri, S. Moeller, H.-D. Nuhn, G. Pile, D. Ratner, J. Rzepiela, D. Schultz, T. Smith, P. Stefan, H. Tompkins, J. Turner, J. Welch, W. White, J. Wu, G. Yocky, and J. Galayda, Nat. Photon. 4, 641 (2010).

3. T. Shintake, H. Tanaka, T. Hara, T. Tanaka, K. Togawa, M. Yabashi, Y. Otake, Y. Asano, T. Bizen, T. Fukui, S. Goto, A. Higashiya, T. Hirono, N. Hosoda, T. Inagaki, S. Inoue, M. Ishii, Y. Kim, H. Kimura, M. Kitamura, T. Kobayashi, H. Maesaka, T. Masuda, S. Matsui, T. Matsushita, X. Maréchal, M. Nagasono, H. Ohashi, T. Ohata, T. Ohshima, K. Onoe, K. Shirasawa, T. Takagi, S. Takahashi, M. Takeuchi, K. Tamasaku, R. Tanaka, Y. Tanaka, T. Tanikawa, T. Togashi, S. Wu, A. Yamashita, K. Yanagida, C. Zhang, H. Kitamura, and T. Ishikawa, Nat. Photon. 2, 555 (2008).

4. T. Ishikawa, H. Aoyagi, T. Asaka, Y. Asano, N. Azumi, T. Bizen, H. Ego, K. Fukami, T. Fukui, Y. Furukawa, S. Goto, H. Hanaki, T. Hara, T. Hasegawa, T. Hatsui, A. Higashiya, T. Hirono, N. Hosoda, M. Ishii, T. Inagaki, Y. Inubushi, T. Itoga, Y. Joti, M. Kago, T. Kameshima, H. Kimura, Y. Kirihara, A. Kiyomichi, T. Kobayashi, C. Kondo, T. Kudo, H. Maesaka, X. Maréchal, T. Masuda, S. Matsubara, T. Matsumoto, T. Matsushita, S. Matsui, M. Nagasono, N. Nariyama, H. Ohashi, T. Ohata, T. Ohshima, S. Ono, Y. Otake, C. Saji, T. Sakurai, T. Sato, K. Sawada, T. Seike, K. Shirasawa, T. Sugimoto, S. Suzuki, S. Takahashi, H. Takebe, K. Takeshita, K. Tamasaku, H. Tanaka, R. Tanaka, T. Tanaka, T. Togashi, K. Togawa, A. Tokuhisa, H. Tomizawa, K. Tono, S. Wu, M. Yabashi, M. Yamaga, A. Yamashita, K. Yanagida, C. Zhang, T. Shintake, H. Kitamura, and N. Kumagai, Nat. Photon. 6, 540 (2012).

5. W. Ackermann, G. Asova, V. Ayvazyan, A. Azima, N. Baboi, J. Bähr, V. Balandin, B. Beutner, A. Brandt, A. Bolzmann, R. Brinkmann, O. I. Brovko, M. Castellano, P. Castro, L. Catani, E. Chiadroni, S. Choroba, A. Cianchi, J. T. Costello, D. Cubaynes, J. Dardis, W. Decking, H. Delsim-Hashemi,
A. Delserieys, G. Di Pirro, M. Dohlus, S. Düsterer, A. Eckhardt, H. T. Edwards, B. Faatz, J. Feldhaus, K. Flöttmann, J. Frisch, L. Fröhlich, T. Garvey, U. Gensch, Ch. Gerth, M. Görler, N. Golubeva, H.-J. Grabosch, M. Grecki, O. Grimm, K. Hacker, U. Hahn, J. H. Han, K. Honkavaara, T. Hott, M. Hüning, Y. Ivanisenko, E. Jaeschke, W. Jalmuzna, T. Jezynski, R. Kammering, V. Katalev, K. Kavanagh, E. T. Kennedy, S. Khodyachykh, K. Klose, V. Kocharyan, M. Körfer, M. Kollewe, W. Koprek, S. Korepanov, D. Kostin, M. Krassilnikov, G. Kube, M. Kuhlmann, C. L. S. Lewis, L. Lilje, T. Limberg, D. Lipka, F. Löhl, H. Luna, M. Luong, M. Martins, M. Meyer, P. Michelato, V. Miltchev, W. D. Möller, L. Monaco, W. F. O. Müller, O. Napieralski, O. Napoly, P. Nicolosi, D. Nölle, T. Nuñez, A. Oppelt, C. Pagani, R. Paparella, N. Pchalek, J. Pedregosa-Gutierrez, B. Petersen, B. Petrosyan, G. Petrosyan, L. Petrosyan, J. Pflüger, E. Plönjes, L. Poletto, K. Pozniak, E. Prat, D. Proch, P. Pucyk, P. Radcliffe, H. Redlin, K. Rehlich, M. Richter, M. Roehrs, J. Roensch, R. Romaniuk, M. Ross, J. Rossbach, V. Rybnikov, M. Sachwitz, E. L. Saldin, W. Sandner, H. Schlarb, B. Schmidt, M. Schmitz, P. Schmüser, J. R. Schneider, E. A. Schneidmiller, S. Schnepp, S. Schreiber, M. Seidel, D. Sertore, A. V. Shabunov, C. Simon, S. Simrock, E. Sombrowski, A. A. Sorokin, P. Spanknebel, R. Spesyvtsev, L. Staykov, B. Steffen, F. Stephan, F. Stulle, H. Thom, K. Tiedtke, M. Tischer, S. Toleikis, R. Treusch, D. Trines, I. Tsakov, E. Vogel, T. Weiland, H. Weise, M. Wellhöfer, M. Wendt, I. Will, A. Winter, K. Wittenburg, W. Wurth, P. Yeates, M. V. Yurkov, I. Zagorodnov, and K. Zapfe, Nat. Photon. 1, 336 (2007).

6. J. Feldhaus, E. L. Saldin, J. R. Schneider, E. A. Schneidmiller, and M. V. Yurkov, Opt. Commun. 140, 341 (1997).

7. G. Geloni, V. Kocharyan, and E. Saldin, DESY10-008 (2010).

8. E. J. Takahashi, T. Kanai, K. L. Ishikawa, Y. Nabakawa, and K. Midorikawa, Phys. Rev. Lett. 101, 253901 (2008).

9. L.-H. Yu, M. Babzien, I. Ben-Zvi, L. F. DiMauro, A. Doyuran, W. Graves, E. Johnson, S. Krinsky, R. Malone, I. Pogorelsky, J. Skaritka, G. Rakowsky, L. Solomon, X. J. Wang, M. Woodle, V. Yakimenko, S. G. Biedron, J. N. Galayda, E. Gluskin, J. Jagger, V. Sajaev, and I. Vasserman, Science 289, 932 (2000).

10. E. Allaria, R. Appio, L. Badano, W. A. Barletta, S. Bassanese, S. G. Biedron, A. Borga, E. Busetto, D. Castronovo, P. Cinquegrana, S. Cleva, D. Cocco, M. Cornacchia, P. Craievich, I. Cudin, G. D'Auria, M. Dal Forno, M. B. Danailov, R. De Monte, G. De Ninno, P. Delgiusto, A. Demidovich, S. Di Mitri, B. Diviacco, A. Fabris, R. Fabris, W. Fawley, M. Ferianis, E. Ferrari, S. Ferry, L. Froehlich, P. Furlan, G. Gaio, F. Gelmetti, L. Giannessi, M. Giannini, R. Gobessi, R. Ivanov, E. Karantzoulis, M. Lonza, A. Lutman, B. Mahieu, M. Milloch, S. V. Milton, M. Musardo, I. Nikolov, S. Noe, F. Parmigiani, G. Penco, M. Petronio, L. Pivetta, M. Predonzani, F. Rossi, L. Rumiz, A. Salom, C. Scafuri, C. Serpico, P. Sigalotti, S. Spampinati, C. Spezzani, M. Svandrlik, C. Svetina, S. Tazzari, M. Trovo, R. Umer, A. Vascotto, M. Veronese, R. Visintini, M. Zaccaria, D. Zangrando, and M. Zangrando, Nat. Photon. 6, 699 (2012).

11. E. Allaria, D. Castronovo, P. Cinquegrana, P. Craievich, M. Dal Forno, M. B. Danailov, G. D'Auria, A. Demidovich, G. De Ninno, S. Di Mitri, B. Diviacco, W. M. Fawley, M. Ferianis, E. Ferrari, L. Froehlich, G. Gaio, D. Gauthier, L. Giannessi, R. Ivanov, B. Mahieu, N. Mahne, I. Nikolov, F. Parmigiani, G. Penco, L. Raimondi, C. Scafuri, C. Serpico, P. Sigalotti, S. Spampinati, C. Spezzani, M. Svandrlik, C. Svetina, M. Trovo, M. Veronese, D. Zangrando, and M. Zangrando, Nat. Photon. 7, 913 (2013). 
12. T. Togashi, E. Takahashi, K. Midorikawa, M. Aoyama, K. Yamakawa, T. Sato, A. Iwasaki, S. Owada, T. Okino, K. Yamanouchi, F. Kannari, A. Yagishita, H. Nakano, M. E. Couprie, K. Fukami, T. Hatsui, T. Hara, T. Kameshima, H. Kitamura, N. Kumagai, S. Matsubara, M. Nagasono, H. Ohashi, T. Ohshima, Y. Otake, T. Shintake, K. Tamasaku, H Tanaka, T. Tanaka, K. Togawa, H. Tomizawa, T. Watanabe, M. Yabashi, and T. Ishikawa, Opt. Express 19, 317 (2011).

13. E. L. Saldin, E. A. Schneidmiller, and M. V. Yurkov, Opt. Commun. 202, 169 (2002).

14. K. Togawa, T. Shintake, T. Inagaki, K. Onoe, T. Tanaka, H. Baba, and H. Matsumoto, Phys. Rev. ST Accel. Beams 10, 020703 (2007).

15. M. Kato, N. Saitoa, T. Tanaka, Y. Morishita, H. Kimura, H. Ohashi, M. Nagasono, M. Yabashi, K. Tono, T. Togashi, A. Higashiya, and T. Ishikawa, Nucl. Instrum. Methods Phys. Res. A 612, 209211 (2009).
16. E. J. Takahashi, Y. Nabekawa, and K. Midorikawa, Appl. Phys. Lett. 84, 4 (2004).

17. E. J. Takahashi, P. Lan, O. D. Mücke, Y. Nabekawa, and K. Midorikawa, Phys. Rev. Lett 104, 233901 (2010).

18. E. J. Takahashi, P. Lan, O. D. Mücke, Y. Nabekawa, and K. Midorikawa, Nat. Commun. 3691, 1 (2013).

19. H. Tomizawa, T. Togashi, K. Ogawa, H. Tanaka, M. Yabashi, S. Matsubara, Y. Okayasu, and T. Ishikawa, in Proceedings of the 2nd International Beam Instrumentation Conference (IBIC2013) MOAL2 (2013).

20. H. Tomizawa, H. Hanaki, and T. Ishikawa, in Proceedings of the 29th International Free Electron Laser Conference 472 (2007).

21. Y. Okayasu, H. Tomizawa, S. Matsubara, N. Kumagai, A. Maekawa, M. Uesaka, and T. Ishikawa, Phys. Rev. ST Accel. Beams 16, 052801 (2013). 